\title{
Visualization Techniques in Smart Grid
}

\author{
Dao Viet Nga, Ong Hang See, Do Nguyet Quang, Chee Yung Xuen, Lai Lee Chee
}

Department of Electronics and Communication Engineering, Universiti Tenaga Nasional, Kejang, Malaysia.

Email: ngachi86@gmail.com,ong@uniten.edu.my,milkydove@yahoo.com,yxche828@gmail.com,doris_lai87@yahoo.com

Received May $13^{\text {th }}$, 2012; revised June $13^{\text {th }}, 2012$; accepted June $20^{\text {th }}, 2012$

\begin{abstract}
Visualization is an established methodology in scientific computing. It has been used in many fields because of its strong capability in large data management and information display. However, its applications in power systems, especially in Smart Grid are still in infancy stage. Besides, while there were a lot of researches working on visualizing data in transmission power system, the study on displaying distribution power system data was limited. Therefore, in this paper, author proposed some techniques to visualize the Smart Grid data at distribution. They are classified in three categories, which are low dimensional techniques, multivariate high dimensional techniques and Geographical Information System (GIS) techniques.
\end{abstract}

Keywords: Smart Grid; Visualization Techniques; Google Earth; GIS; QGIS, AMI; SCADA; Spatial; Temporal; Animation

\section{Introduction}

A large number of novel information visualization techniques have been developed over the past decade, allowing visualizations of larger and more complex such as multidimensional, multivariate, multi temporal, and spatial data sets. They are listed in many research publications [1-5].

In earlier researches, there are a lot of researches which had been worked on visualizing data in transmission power system [6-10]. Some traditional methods, such as graph, histogram, bar chart, pie chart, single line diagram, are mandatory for visualizing power data. Besides, Geographic visualization has become quite famous technique in displaying data in transmission grid, such as contour [11-14], GDV (graphical data view) [15], Green Grid [16]. AREVA's Energy Management System (EMS) [17] and Power World's Simulator [10] are two widely used visualization tools in this industry. There are also some research works on animated visualization for power grid data such line flow [18] or power flow [19]. However, the use of high dimensional techniques has not been applied in this area.

Nevertheless, the numbers of research on displaying distribution power system data were still limited. Yixin Cai in his research proposed GIS as a technique for visualizing fault locations in distribution system based on spatial-temporal dataset [20].

In this paper, proposed techniques to visualize the Smart Grid distribution data are classified in three categories, which are low dimensional techniques, multivari- ate high dimensional techniques and Geographical Information System (GIS). Traditional methods consist of some traditional techniques such as single line diagram, real-time 2D chart and 3D surface with contour. Multivariate high dimensional techniques include parallel coordinate, scatter diagram and Andrew curve. Spatial analysis and spatial-temporal analysis are two geographical techniques that are used in GIS for analyze and visualize Smart Grid data. These can be shown in Figure 1.

The visualize tools used in this paper are Quantum GIS (QGIS) which is open source GIS software, Google Earth, Microsoft Chart and Matlab.

\section{AMI and SCADA}

This paper will focus only on AMI and SCADA data in Smart Grid. Below are some AMI data which were used by earlier researches:

- Voltage/Current/Power consumption [21,22].

- Outage [21].

- Peak demand signal [23].

- Pricing information [23].

- Energized state of AMI devices [19].

RTU/SCADA data was also mentioned in some previous paper, such as:

- Power/Current on distribution feeder and Voltage at Substation bus [24].

- Real-time measurements of voltage, current, real power, reactive power, breaker status, transformer taps, etc. from substation RTUs and other nodes of the distribution system [24]. 
This project concentrates on Uniten-TNBR Smart Grid Testbed. There are 16 substations located in this area which divided into 3 main substations such as TNB SSU, TNB SSU1, and TNB SSU2. Each substation is divided into smaller unit called PE. The detail of AMI and SCADA network diagram is shown in Figure 2.

\section{Traditional Visualization Techniques}

\subsection{Single Line Diagram}

The operators use single line diagrams to get an overview of the AMI/SCADA network system. Single line diagrams display the interconnection between substations and some critical parameters such as line status, AMI energized status. Hence, the diagram allows the operators to have a macro level view of the system as shown in Figure 3.

\subsection{Real Time 2D Bar Chart}

Bar chart is one of traditional techniques to visualize data in many fields. However, instead of static data, dynamic data is displayed in form of bar chart. The x-axis represents number of data points while the y-axis represents total power consumption of all substations, as shown in Figure 4. Since a data point was added in every 5 minutes and the maximum of point displaying in $\mathrm{x}$-axis are 200, the chart can visualize the changing of total power consumption over 1000 minutes time interval. When the new data point adds to the chart, the data at the most left of the horizontal axis will be removed.

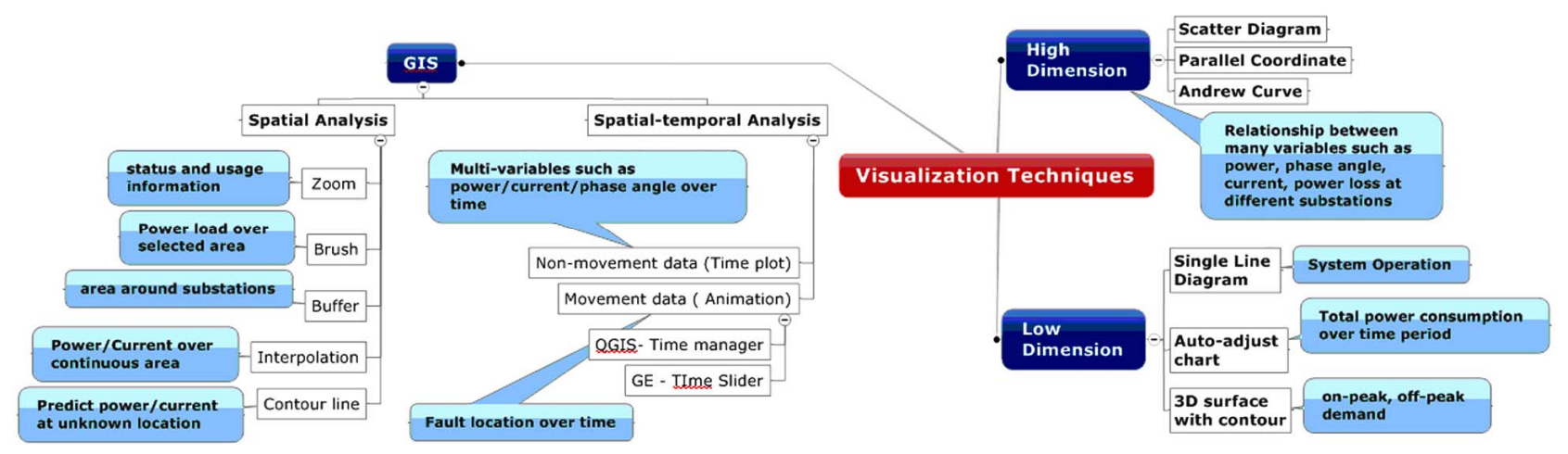

Figure 1. Visualization techniques in Smart Grid.

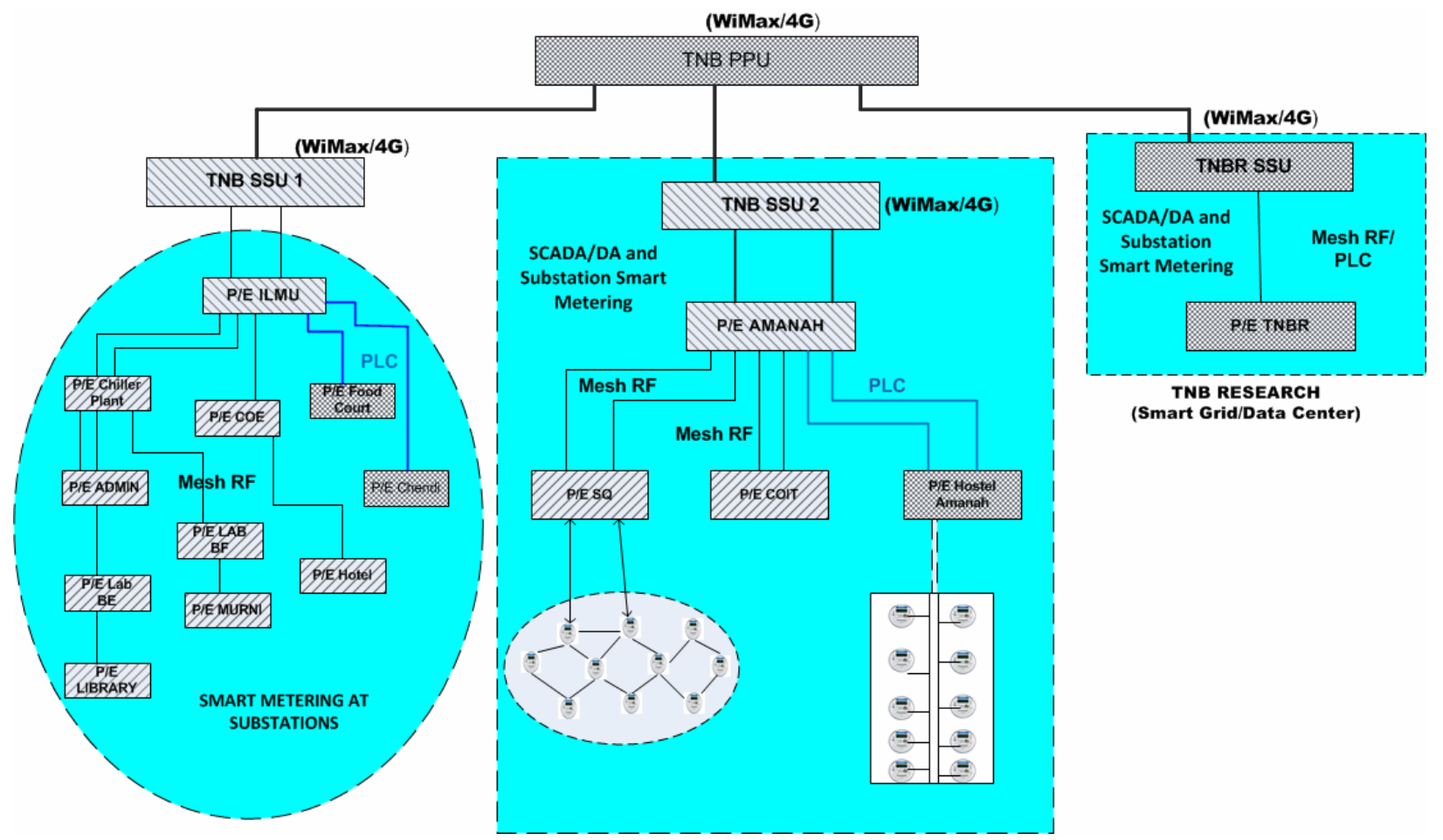

Figure 2. AMI and SCADA network diagram for Uniten-TNBR smart grid testbed. 


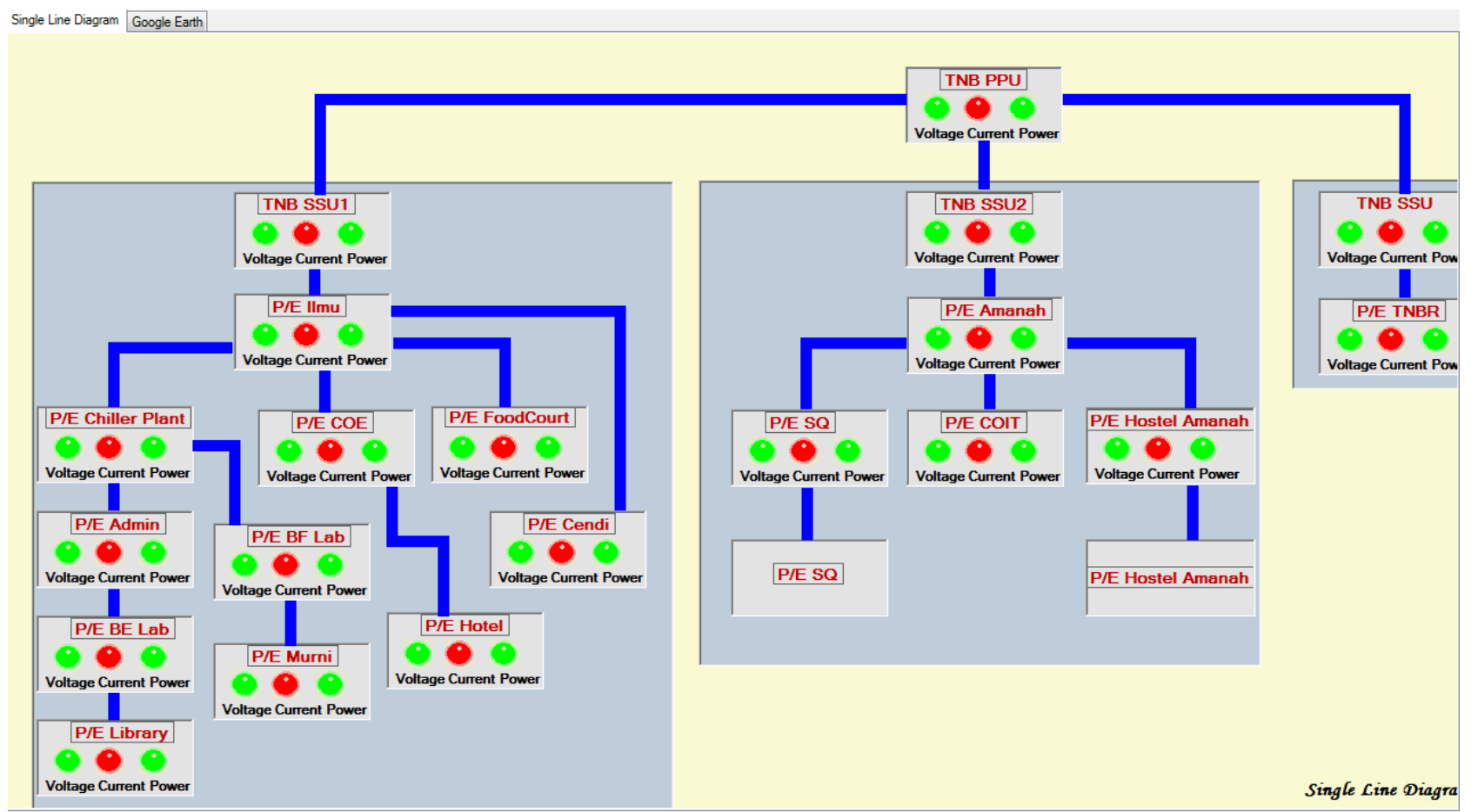

Figure 3. Single line diagram.

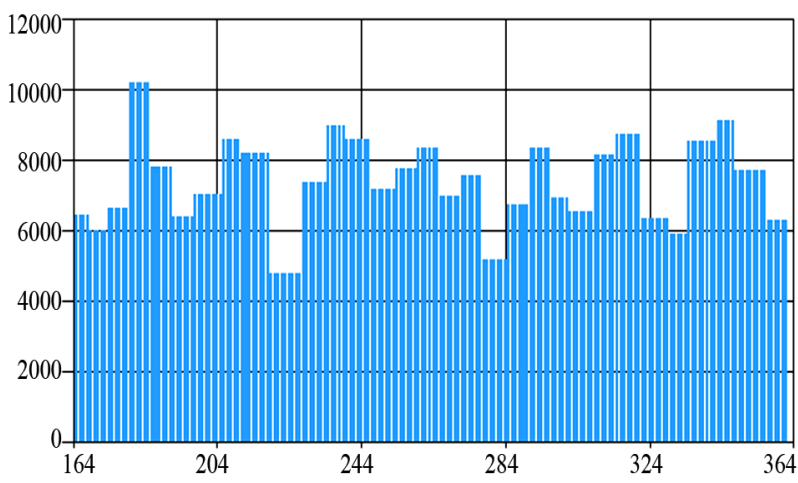

Figure 4. Real time bar chart.

\subsection{D Surface with Contour [14]}

The grid operators use the 3-D displays to visualize the power consumption or load in every 1hour interval over a month period. A 3-D illustration of the demand in the studied area is shown in the Figure 5. One hour intervals are shown on $\mathrm{x}$-axis from 1 to 24 and the dates of month are shown on the $y$-axis from 1 to 31 . The power magnitude is represented on the z-axis. The color contouring is applied to enhance the display, as shown in the color bar at the right of the plot. As we can see from the figure, the peak (morning and evening) and off-peak (day and night) variation over a month can be clearly brought out.

To visualize the change of power load over a year, the $\mathrm{x}$-axis can represent the dates of months [1-31] while the $\mathrm{y}$-axis can represent the months in years [1-12].

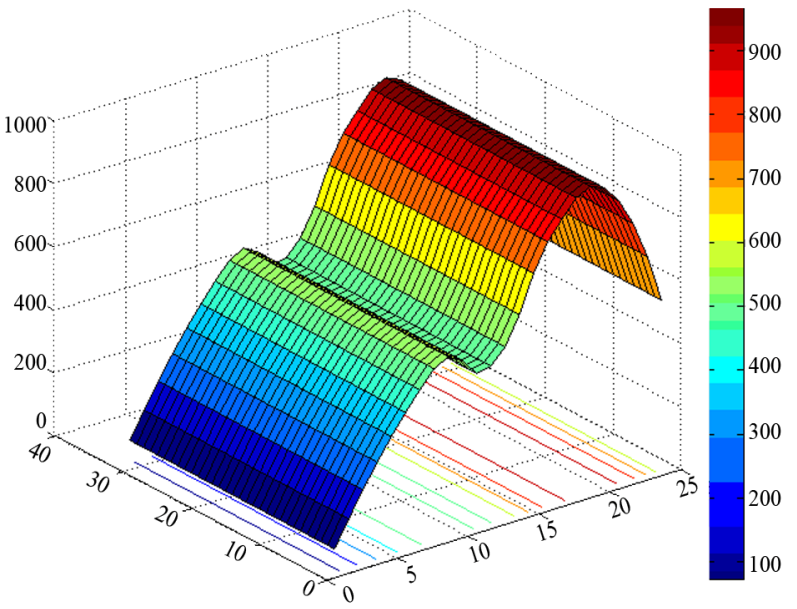

Figure 5. 3D surface with contour.

\section{Multivariate High Dimensional Visualization}

Many statistical analyses involve only two variables: a predictor variable and a response variable. Such data are easy to visualize using 2D scatter plots, bivariate histograms, box plots and so on. It's also possible to visualize trivariate data with 3D scatter plots or 2D scatter plots with a third variable encoded with color. However, many datasets involve a larger number of variables, making direct visualization more difficult. There are some methods to visualize high-dimensional data, such as scatter diagram, parallel coordinate, and Andrew curve. This can 
be achieved by using Matlab Statistic toolbox.

This paper illustrates multivariate visualization by using five variable such as power consumption (kWh), load (in $\mathrm{kW}$ ), power factor, current (in A), and power loss (in $\mathrm{kW}$ ). The name of substation is used to group observations, e.g. TNB SSU (TNBR), TNB SSU1 (Ilmu) and TNB SSU2 (Amanah).

\subsection{Scatter Diagram}

Viewing slices through lower dimensional subspaces is one way to partially work around the limitation of two or three dimensions. This can display an array of all the bi-variate scatter plots between five variables in the project, along with a uni-variate histogram for each variable.

In Figure 6, the points in each scatter plot are colorcoded by different substations: red for TNB SSU (TNBR), green for TNB SSU1 (Ilmu), and blue for TNB SSU2 (Amanah). This array of plots makes it easy to pick out patterns in the relationships between pairs of variables. However, there may be important patterns in higher dimensions, and those are not easy to recognize in this plot.

\subsection{Parallel Coordinate}

The scatter plot matrix only displays bivariate relationships. However, there are other alternatives that display all the variables together, allowing operator to investigate higher-dimensional relationships among variables. The most straight-forward multivariate plot is the parallel coordinates plot. In this plot, the coordinate axes are all laid out horizontally, instead of using orthogonal axes as in the usual Cartesian graph. Each observation is represented in the plot as a series of connected line segments. Similar to scatter plot, in this paper, this plot shows colour observations by group of substations, as shown in Figure 7.

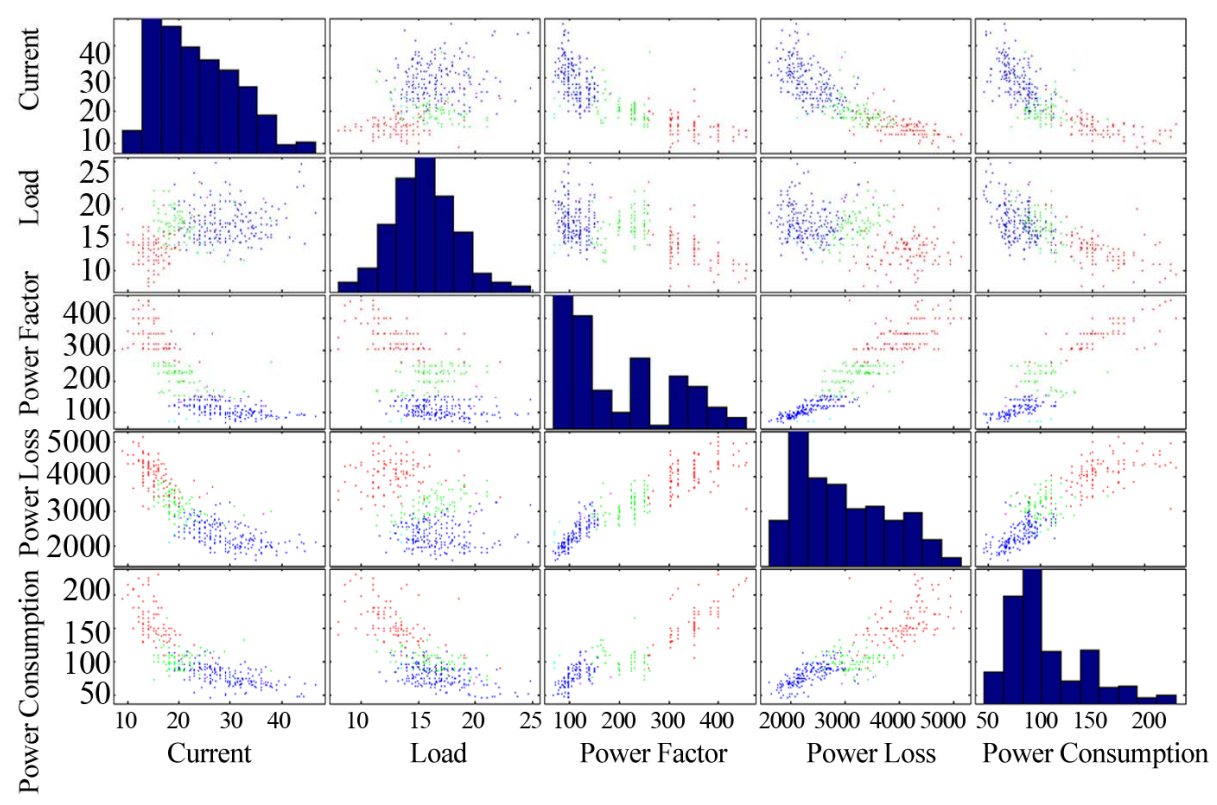

Figure 6. Scatter diagram.

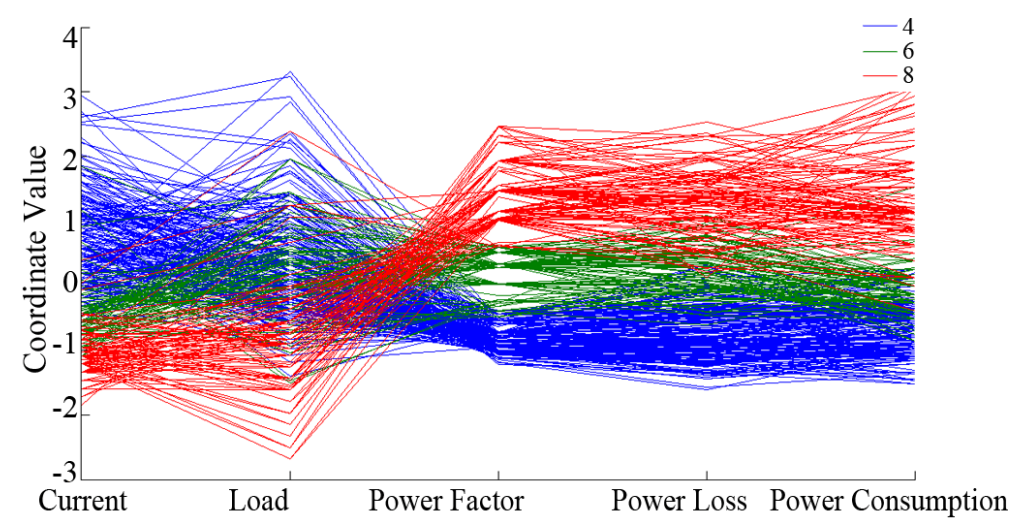

Figure 7. Parallel coordinate. 
The horizontal direction in this plot represents the coordinate axes, and the vertical direction represents the data. Each observation consists of measurements on five variables, and each measurement is represented as the height at which the corresponding line crosses each coordinate axis. Because the five variables have widely different ranges, this plot was made with standardized values, where each variable has been standardized to have zero mean and unit variance. With the colour coding, the graph shows that TNB SSU (TNBR) typically have low values for power loss and current but high values for power factor, load and power consumption. In the opposite, TNB SSU2 (Amanah) have high value for power loss and current, and high values for power factor, load and power consumption.

As we can see from Figure 7, it is difficult to recognize the relationship between 5 variables even with colour coding by group. Therefore, one way to simplify the graph is by making a parallel coordinates plot where only the median and quartiles (25\% and $75 \%$ points) for each group are shown (Figure 8). This makes the typical dif- ferences and similarities among groups easier to distinguish. On the other hand, it may be the outliers for each group that are most interesting, and this plot does not show them at all.

\subsection{Andrew Curve}

Another similar type of multivariate visualization is the Andrews plot. This plot represents each observation as a smooth function over the interval $[0,1]$. Each function is a Fourier series, with coefficients equal to the corresponding observation's values. In this project, the series has five terms: a constant, two sine terms with periods 1 and $1 / 2$, and two similar cosine terms (Figure 9).

There's a distinct difference between groups at $t=0$, indicating that the first variable, current, is one of the distinguishing features between 3 substation groups. More interesting is the difference between the three groups at around $t=1 / 3$. Plugging this value into the formula for the Andrews plot functions, a set of coefficients that define a linear combination of the variables that distinguishes between groups are obtained.

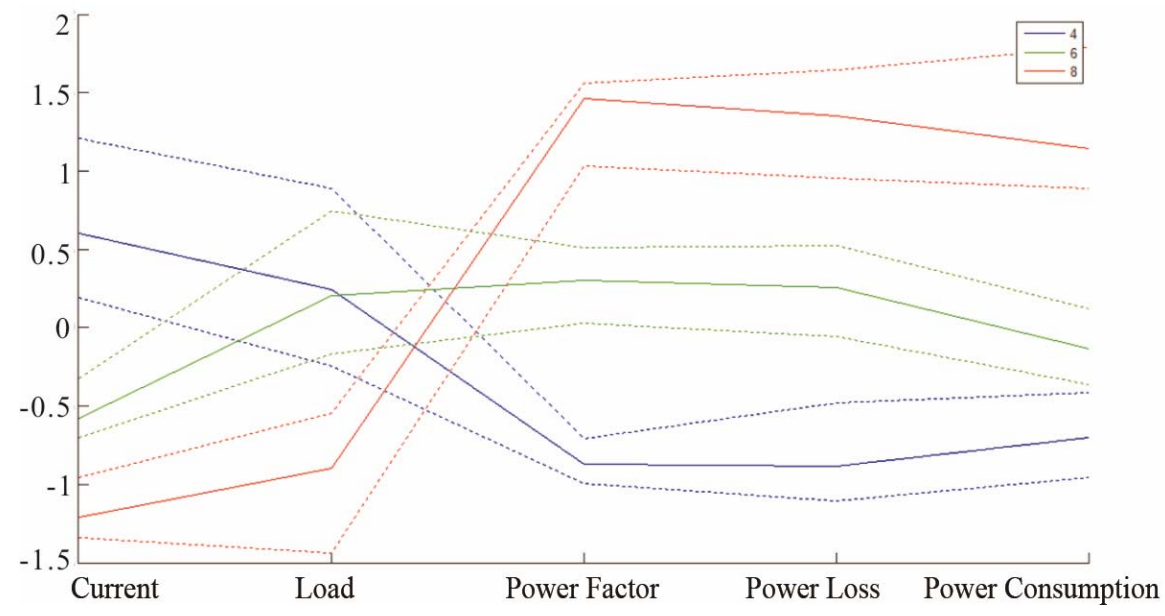

Figure 8. Simplified parallel coordinate.

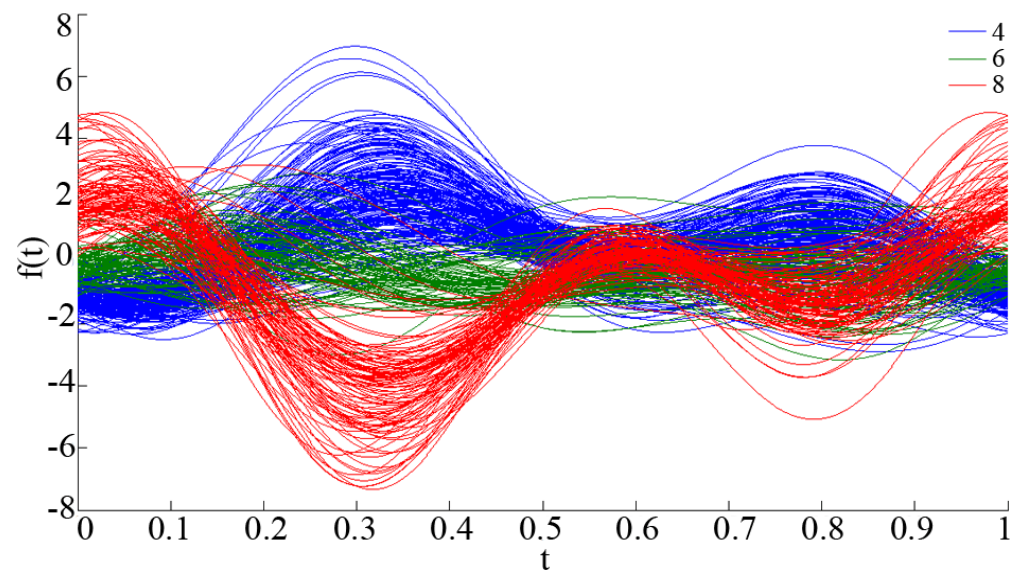

Figure 9. Andrew curve. 


$$
\begin{gathered}
t=\frac{1}{3} ; \\
\frac{1}{\sqrt{2}} \sin (2 \pi t) \cos (2 \pi t) \sin (4 \pi t) \cos (4 \pi t) \\
=0.7071 \quad 0.8660 \quad-0.5000-0.8660 \quad-0.5000
\end{gathered}
$$

From these coefficients and the graph, it can seen that one way to distinguish TNB SSU (red with negative value) from TNB SSU2 (blue with positive value) is that the latter have higher values of current and power loss, and lower values of power consumption, load, and power factor, while the latter have the opposite. That's the same conclusion we drew from the parallel coordinates plot.

\section{Geographic Information System}

\subsection{Spatial Analysis}

Spatial analysis uses spatial information to extract new and additional meaning from GIS data. GIS Applications normally have spatial analysis tools for feature statistics (e.g. how many vertices make up this polyline) and geoprocessing tools such as buffer, intersect, union, symmetric difference and so on [25].

\subsubsection{Zoom and Brush}

Each AMI/SCADA device is represented by a point or a placemark which can be created by using Google Earth API, The detail information of each substation such as power consumption, voltage, current from are displayed on AMI balloon. Real-time measurements of volt- age, current, real power, reactive power, transformer taps from SCADA are displayed in balloon callout corresponding to each SCADA placemark. The color of each placemark represents the energized state of AMI and breaker status of SCADA, e.g. green is energized and red is not (Figure 10).

As shown in Figure 10, the left pane of the GUI is the list of all the 16 substations. Clicking on any one of these will result in flying into each point where detail parameters are displayed.

Another benefit of this application is to allow user select the region of interest by dragging the mouse in GE browser to define the area and subsequently displaying the total power as new window message, as shown in Figure 11.

\subsubsection{Buffer Analysis}

Quantum GIS (QGIS) is a powerful Open Source GIS. The software allows users to design their own map with custom features (represented by point, line, and polygon) [25].

Spatial analysis uses spatial information to extract new and additional meaning from GIS data. GIS Applications normally have spatial analysis tools for feature statistics (e.g. how many vertices make up this polyline) and geoprocessing such as buffer, symmetric, difference, and so on [25].

Buffering usually creates two areas: one area is within a specified distance to selected features, is called the buffer zone. Area which is beyond the specified distances

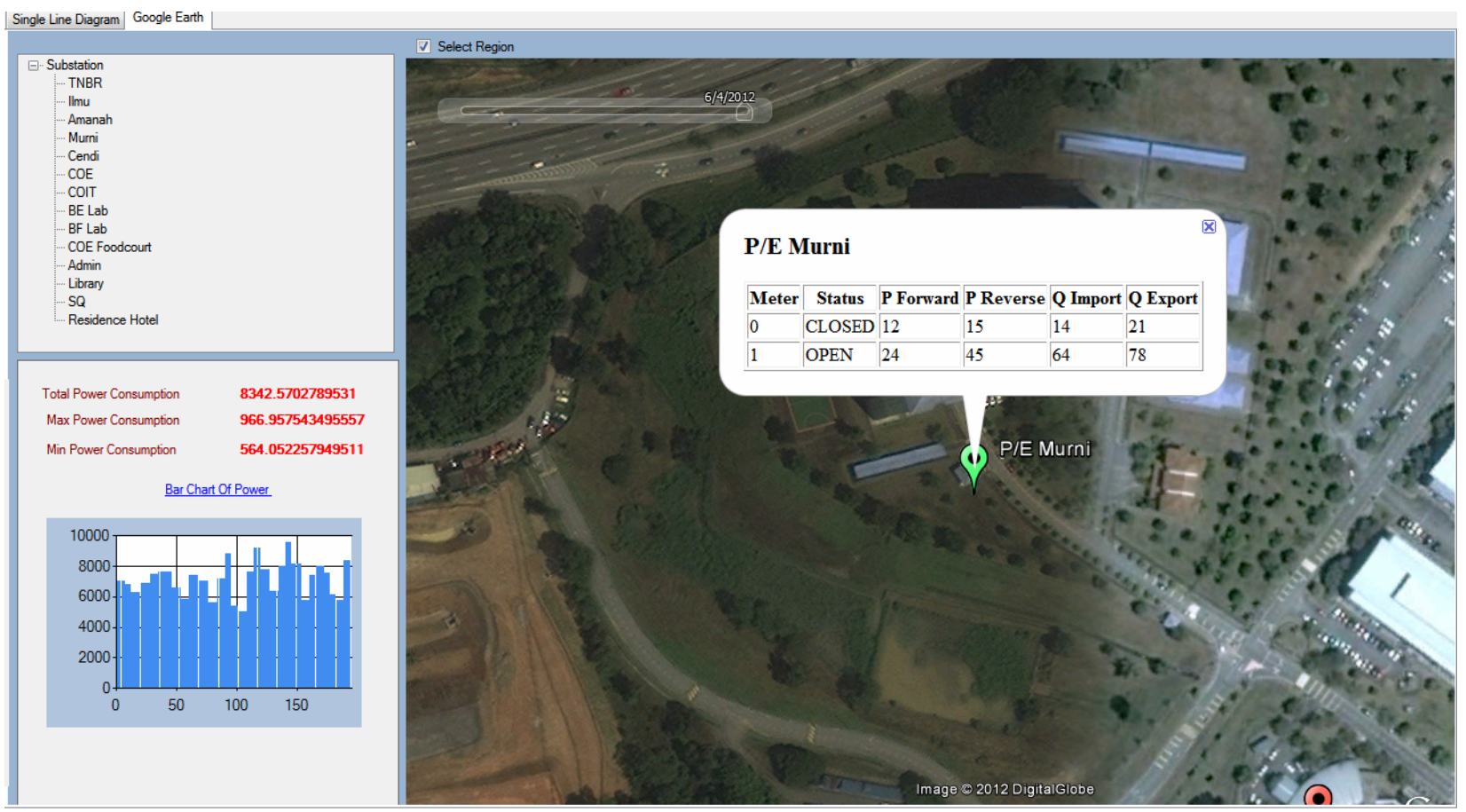

Figure 10. Zoom. 


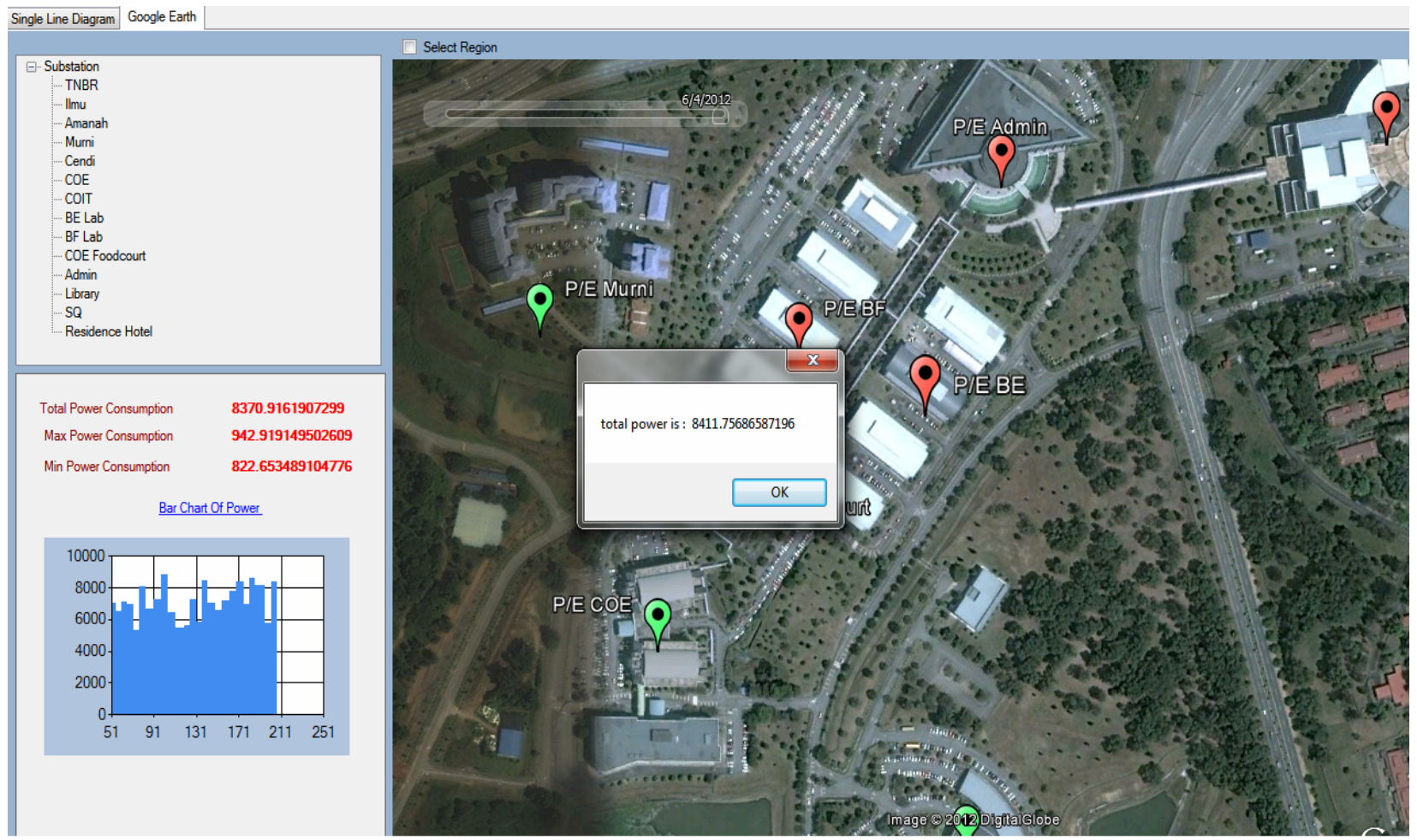

Figure 11. Brush.

is none-buffer zone [26].

In a GIS Application, buffer zones are always represented as vector polygons enclosing other polygon, line or point features as shown in Figure 12.

Buffer zones often have dissolved boundaries so that there are no overlapping areas between the buffer zones. In some cases though, it may also be useful to remain boundaries of buffer zones, so that each buffer zone is a separate and the overlapping areas can be identified.

In this project, the point features in GIS are designed to represent the power substation in Smart Grid. The buffer zone of Smart Grid substation point vector with distance of 0.001 map unit corresponding to Coordinate Reference System (CRS), e.g. WGS 84 is shown in Figure 13.

\subsubsection{Interpolation Analysis}

Spatial interpolation is the process of using points with known values to estimate values at other unknown points [26]. Because of high cost and limited resources, data collection is usually conducted only in a limited number of selected point locations. In GIS, spatial interpolation of these points can be applied to create a raster surface with estimates made for all raster cells. In order to generate a continuous map, a suitable interpolation method has to be used to estimate the values at those locations where no samples or measurements were taken. The results of the interpolation analysis can then be used for analyze the whole area and for modeling.

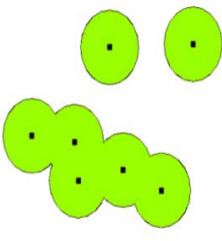

(a)

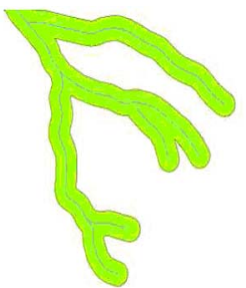

(b)

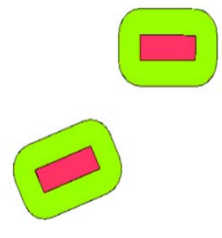

(c)
Figure 12. Buffering vector points, polylines and polygons [26]. (a) A buffer zone around vector points; (b) A buffer zone around vector polylines; (c) A buffer zone around vector polygons.

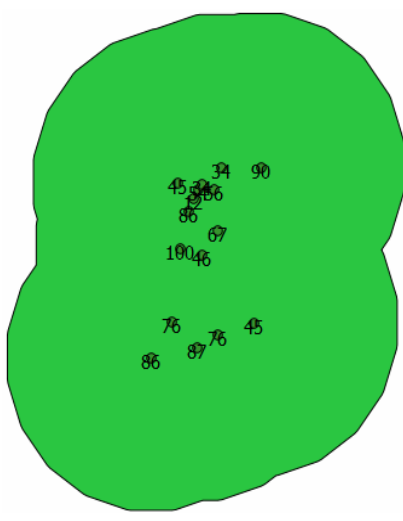

(a)

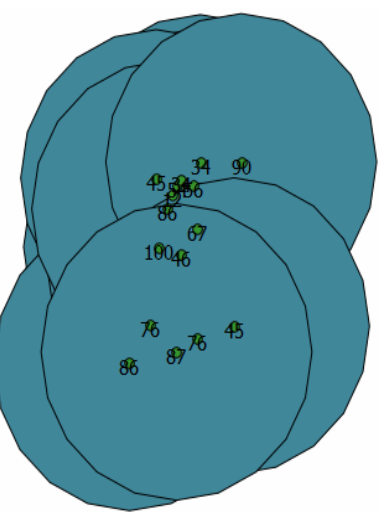

(b)
Figure 13. Buffer zone (a) With dissolve feature; (b) Without dissolve feature. 
There are many interpolation methods. In this paper, two widely used interpolation methods called Inverse Distance Weighting (IDW) and Triangulated Irregular Networks (TIN) are presented [26].

The application of interpolation in Smart Grid is to visualize the contour demand power, current or billing information in whole Uniten-TNBR area based on these values at 16 substations (Figure 14).

\subsubsection{Contour Line}

Contour lines are lines drawn on a map connecting points of equal elevation [27]. Contouring has been used very effectively to represent spatially distributed continuous data e.g., temperature. However, power system data is not spatially continuous for example, power or current exist only at specified substation. Thus to use contouring for power system data, virtual values must be assigned to the entire region. The operators have used contouring to

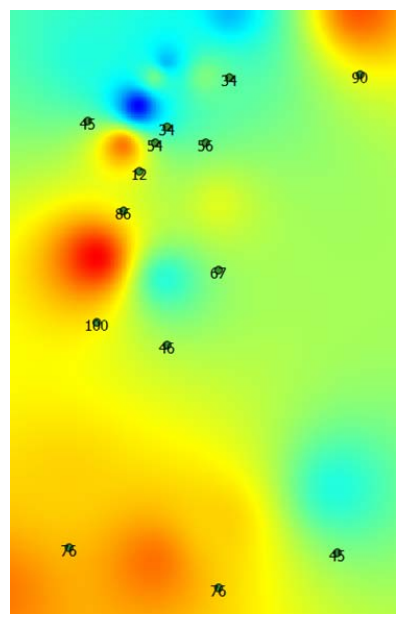

(a)

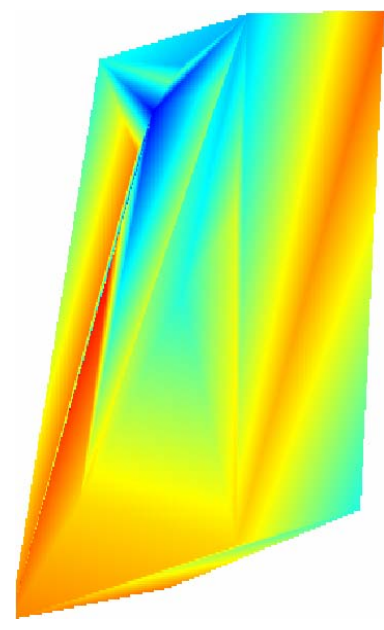

(b)
Figure 14. Interpolation using (a) IDW; (b) TIN.

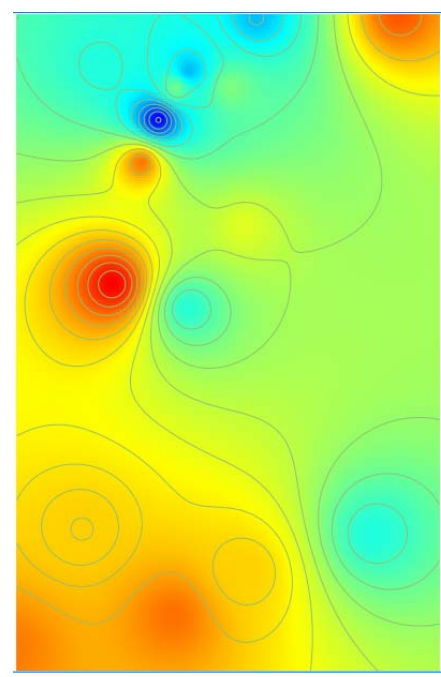

Figure 15. Contour line. represent the load power in the system. An illustration showing load power for the studied region is shown in Figure 15. From the contour line, we can predict quite accurately the load power at different desired location.

\subsection{Spatial-Temporal Analysis}

Almost everything in the world changes over time. It is necessary to incorporate time into geographical information systems. This is known as spatio-temporal analysis which is capable of handling temporal as well as spatial information. This greatly expands current GIS applications and allows new information to be obtained [28-31].

Spatio-temporal data can be classified into two categories: movement and static data [1]. Several visualisations exist for movement data over time including: maps with time stamped objects [30], Space-Time cube, Multivariable- and PCP-Time-Cube [31]. On the other hand, pencil and helix glyphs [32], animation techniques [26] allow displaying changes over time for specific locations (static).

\subsubsection{Time Plot}

Time plot in MultiView plugin in QGIS allow users to plot multivariate and multi-temporal data at different locations. The $\mathrm{x}$-axis of the plot represents the date time in dd-mm-yy hr:min:sec format. The y-axis represents the value of selected variables in the left panel. The different variables are displayed by different color for better visual. For example, the variation of power over time line graph is colored by red while the variation of power loss over time is colored by blue, as shown in Figure 16. The location is specified by the mouse position in the map canvas with raster data. The raster data are created by applying interpolation for different fields in vector data. For instance, substation, power consumption, and phase angle are three fields/variables in Uniten-SG vector layer. As described above, there are 16 substations in this project with different locations. To display the change of power consumption of each substation over time, this point vector layer must be converted to raster data using interpolation method for power field. Each raster data is corresponding to a specific time. If we want to display power over 24 hours with time resolution is 1 hour, then the time step is 24 , and the numbers of raster data needed are 24. This plug-in allows user to set the starting time as well. This procedure can be applied for all other variables in the vector layer. After raster data are created, it must be enable to display in map canvas. The movement of mouse will decide the location of variables which users want to visualize in the plot.

The advantage of this time plot is the ability to visualize multi variables at different locations versus time in one graph. Since each field has their own scale, it must 
be normalized before displaying in the plot. However, the disadvantage of this plug-in is not utilizing the time stamp variable. If the users want to display data over long time period, the numbers of generated raster data will be very big.

\subsubsection{Animation}

While time plot is useful for visualizing static (no movement) data versus time, animation is used for displaying the movement data versus time. In Smart Grid, this could help to show the change of fault locations over time period. Animation can be achieved by using QGIS with Timer Manager plug-in (Figure 17) or Google Earth with Time Slider (Figure 18).

The Time Manager dock contains some useful features which allow users to load the vector layer with timestamp is one of the variables. The start and stop time can be setting as well. The button for auto play and pause is another feature to help users view the animation as video. The users can also manually forward or backward to

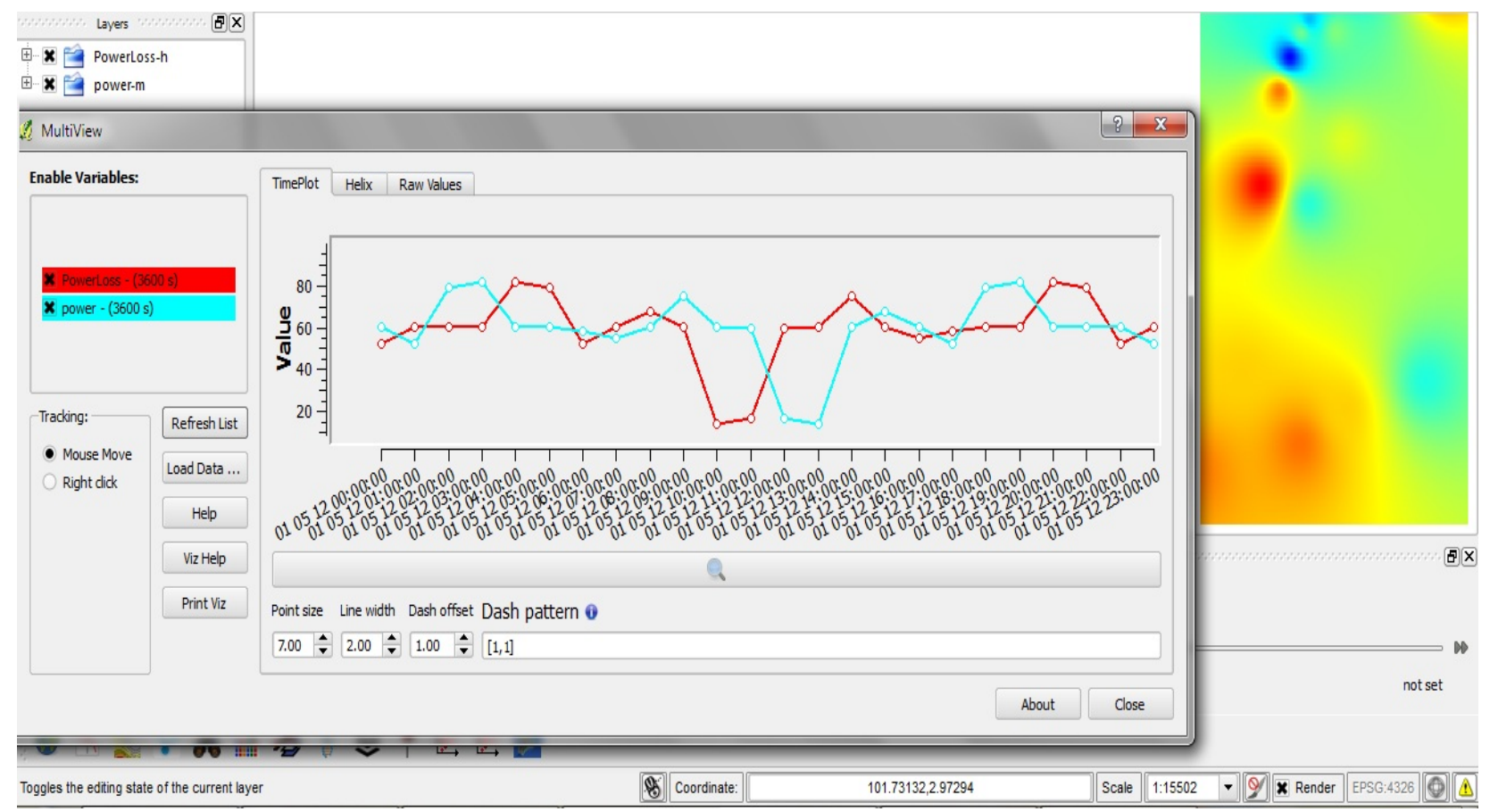

Figure 16. Time plot.

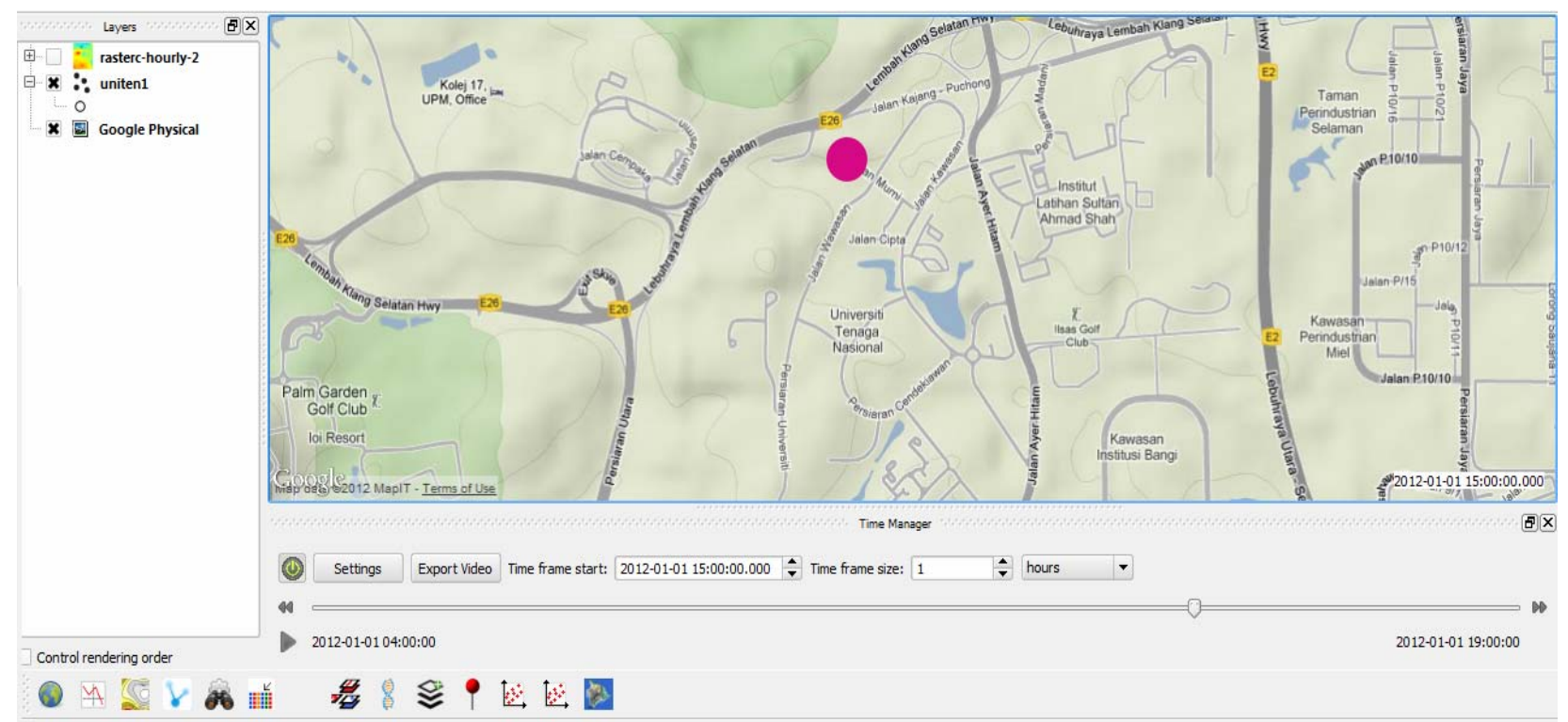

Figure 17. Animation with QGIS. 


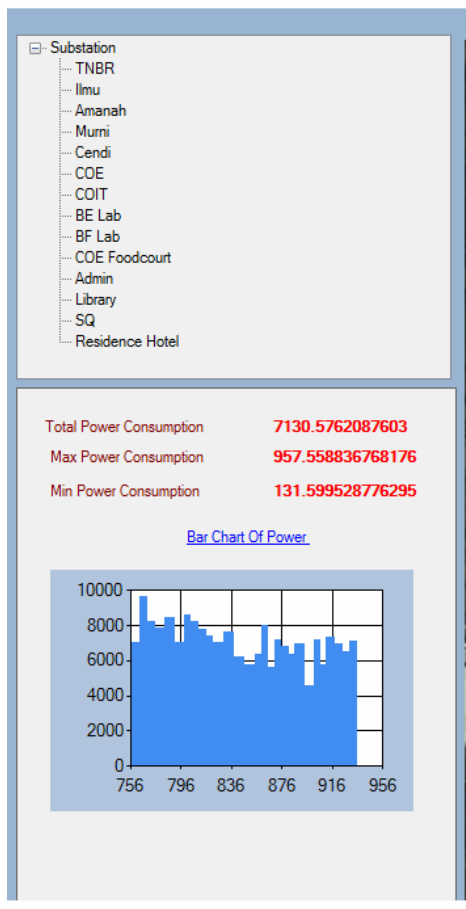

their desired time.

The similar result can be display in Google Earth by using Time Slider feature. This allows users to visualize the historical image over period time, which can apply for viewing the changing of fault location in Smart Grid.

\section{Acknowledgements}

I would like to thank my supervisor Associate Professor Dr Ong Hang See for his guidance and TNBR staffs for network design information.

\section{REFERENCES}

[1] M. Bernasocchi, "Visualizing Multivariate Spatial-Temporal Data,” M.Sc. Thesis, University of Zurich, Zurich, 2011.

[2] H. L. Jin and H. J. Liu, "Research on Visualization Techniques in Data Mining," International Conference on Computational Intelligence and Software Engineering, Wuhan, 11-13 December 2009, pp. 1-3. doi:10.1109/CISE.2009.5366364

[3] D. A. Keim, "Visual Exploration of Large Data Sets," Communications of the ACM, Vol. 44, No. 8, 2001, pp. 38-44. doi:10.1145/381641.381656

[4] D. A. Keim, "Information Visualization and Visual Data Mining," IEEE Transactions on Visualization and Computer Graphics, Vol. 8, No. 1, 2002, pp. 1-8. doi: $10.1109 / 2945.981847$

[5] M. C. F. de Oliveira and H. Levkowitz, "From Visual Data Exploration to Visual Data Mining: A Survey," IEEE Transactions on Visualization and Computer
Graphics, Vol. 9, No. 3, 2003, pp. 378-394. doi:10.1109/TVCG.2003.1207445

[6] T. J. Overbye and J. D. Weber, "Visualization of Power System Data," Proceedings of the 33rd Annual Hawaii International Conference on System Sciences, Maui, 4-7 January 2000, p. 7. doi:10.1109/HICSS.2000.926744

[7] J. D. Weber and T. J. Overbye, "Voltage Contour for Power System Visualization," IEEE Transactions on Power Systems, Vol. 15, No. 1, pp. 404-409. doi:10.1109/59.852151

[8] T. J. Overbye, "Power System Visualization," Automation of Electric Power Systems, Vol. 29, No. 16, 2005.

[9] T. J. Overbye, A. P. Meliopoulos, D. A. Wiegmann and G. J. Cokkinides, "Visualization of Power Systems and Components,” Power Systems Engineering Research Center, Ithaca, 2005.

[10] T. J. Overbye and J. D. Weber, "New Methods for Visualization of Electric Power System Information," IEEE Symposium on Information Visualization, Salt Lake City, 9-10 October 2000, pp. 131-136.

[11] T. J. Overbye and D. A. Wiegmann, "Reducing the Risk of Major Blackouts through Improved Power System Visualization,” 15th Power System Computation Conference, Liege, 22-26 August 2005, 8 p.

[12] T. J. Overbye, “The Role of Power System Visualization in Enhancing Power System Security,” In: S. C. Savulescu, Ed., Real-Time Stability in Power Systems: Techniques for Early Detection of the Risk of Blackout, Springer, New York, 2005, pp. 293-314.

[13] T. J. Overbye, "Transmission System Visualization for the Smart Grid," Power Systems Conference and Exposition, Atlanta, 15-18 March 2009, pp. 1-2. 
[14] R. Klump, D. Schooley and T. Overbye, "An Advanced Visualization Platform for Real-Time Simulations,” 14th Power System Computation Conference, Sevilla, 24-28 June 2002, 8 p.

[15] T. J. Overbye, E. M. Rantanen and S. Judd, "Electric Power Control Center Visualization Using Geographic Data Views,” Bulk Power System Dynamics and Control-VII. Revitalizing Operational Reliability, 2007 iREP Symposium, Charleston, 19-24 August 2007, pp. 1-8. doi:10.1109/TVCG.2008.197

[16] P. C. Wong, K. Schneider, P. Mackey, H. Foote, G. Chin, R. Guttromson and J. Thomas, "A Novel Visualization Technique for Electric Power Grid Analytics,” IEEE Transactions on Visualization and Computer Graphics, Vol. 15, No. 3, 2009, pp. 410-423.

[17] AREVA T \& D Energy Management Systems, 2008. Available from: http://www.areva-td.com

[18] Y. Sun and T. J. Overbye, "Visualization for Power System Contingency Analysis Data," IEEE Transactions on Power Systems, Vol. 19, No. 4, 2004, pp. 1859-1866. doi:10.1109/TPWRS.2004.836193

[19] E. Boardman, "The Role of Integrated Distribution Management Systems in Smart Grid Implementations," Power and Energy Society General Meeting, Minneapolis, 25-29 July 2010, pp. 1-6.

[20] Y. X. Cai and M.-Y. Chow, "Exploratory Analysis of Massive Data for Distribution Fault Diagnosis in Smart Grids,” Power \& Energy Society General Meeting, Calgary, 26-30 July 2009, pp. 1-6. doi:10.1109/PES.2009.5275689

[21] H. Tram, "Technical and Operation Considerations in Using Smart Metering for Outage Management," Transmission and Distribution Conference and Exposition, Chicago, 21-24 April 2008, pp. 1-3. doi:10.1109/TDC.2008.4517273

[22] J. Triplett, S. Rinell and J. Foote, "Evaluating Distribution System Losses Using Data from Deployed AMI and GIS Systems," Rural Electric Power Conference, Orlando, 16-19 May 2010, pp. C1-C8. doi:10.1109/REPCON.2010.5476204

[23] D. Y. R. Nagesh, J. V. V. Krishna and S. S. Tulasiram,
“A Real-Time Architecture for Smart Energy Management,” Innovative Smart Grid Technologies, Gaithersburg, 19-21 January 2010, pp. 1-4.

[24] S. Nunoo and A. K. Ofei, "Distribution Automation (DA) Using Supervisory Control and Data Acquisition (SCADA) with Advanced Metering Infrastructure (AMI),” 2010 IEEE Conference on Innovative Technologies for an Efficient and Reliable Electricity Supply, Waltham, 27-29 September 2010, pp. 454-458. doi:10.1109/CITRES.2010.5619797

[25] D. J. Maguire, M. Batty and M. F. Goodchild, “GIS, Spatial Analysis, and Modelling,” ESRI Press, Redlands, 2005.

[26] T. Sutton, O. Dassau and M. Sutton, "A Gentle Introduction to GIS," Chief Directorate: Spatial Planning \& Information, Eastern Cape, 2009.

[27] http://raider.mountunion.edu/ mcnaugma/topographic\%2 0maps/contour.htm

[28] P. Compieta, S. Di Martino, M. Bertolotto, F. Ferrucci and T. Kechadi, "Exploratory Spatio-Temporal Data Mining and Visualization,” Journal of Visual Languages \& Computing, Vol. 18, No. 3, 2007, pp. 255-279. doi:10.1016/j.jvlc.2007.02.006

[29] M. Weber, M. Alexa and W. Muller, "Visualizing TimeSeries on Spirals,” 2001 IEEE Symposium on Information Visualization, San Diego, 22-23 October 2001, pp. 7-13. doi:10.1109/INFVIS.2001.963273

[30] A. Kjellin, L. W. Pettersson, S. Seipel and M. Lind, "Evaluating 2D and 3D Visualizations of Spatiotemporal Information," ACM Transactions on Applied Perception, Vol. 7, No. 3, 2010, Article ID: 19.

[31] X. Li and M.-J. Kraak, "New Views on Multivariable Spatiotemporal Data: The Space Time Cube Expanded,” International Symposium on Spatio-Temporal Modeling, Spatial Reasoning, Analysis, Data Mining and Data Fusion, Vol. 36, 2005, pp. 199-201.

[32] C. Tominski, P. Schulze-Wollgast and H. Schumann, “3D Information Visualization for Time Dependent Data on Maps," Proceedings of the 9th International Conference Information Visualization, Cambridge, 6-8 July 2005, pp. 175-181. doi:10.1109/IV.2005.3 